\title{
Anti-HEV IgG Avidity Testing: Utility for Diagnosing Acute and Resolved Genotype 3 Infections
}

\author{
Claudia Minosse ${ }^{1}\left(\mathbb{D}\right.$, Daniele Lapa $^{1}$, Antonio Coppola ${ }^{1}$, Federica Rapagna ${ }^{2}$, Gianpiero D'Offizi ${ }^{3}$, Chiara Taibi ${ }^{3}$, \\ Raffaella Lionetti $^{3}$, Maria Rosaria Capobianchi ${ }^{1}$, Fiona McPhee ${ }^{4}$ and Anna Rosa Garbuglia ${ }^{1, *(D)}$ \\ 1 Laboratory of Virology, "Lazzaro Spallanzani" National Institute for Infectious Diseases, IRCCS, \\ 00149 Rome, Italy; claudia.minosse@inmi.it (C.M.); daniele.lapa@inmi.it (D.L.); \\ antonio.coppola@inmi.it (A.C.); maria.capobianchi@inmi.it (M.R.C.) \\ 2 Eurospital SpA, Via Flavia 122, 34147 Trieste, Italy; frapagna@eurospital.it \\ 3 Hepatology and Infectious Diseases Unit, "Lazzaro Spallanzani" National Institute for Infectious Diseases, \\ IRCCS, 00149 Rome, Italy; gianpiero.doffizi@inmi.it (G.D.); chiara.taibi@inmi.it (C.T.); \\ raffaella.lionetti@inmi.it (R.L.) \\ 4 Bristol-Myers Squibb, Cambridge, MA 02142, USA; fiona.mcphee@bms.com \\ * Correspondence: argarbuglia@iol.it; Tel.: +39-0655170692; Fax: +39-065594555
}

Citation: Minosse, C.; Lapa, D.; Coppola, A.; Rapagna, F.; D'Offizi, G.; Taibi, C.; Lionetti, R.; Capobianchi, M.R.; McPhee, F.; Garbuglia, A.R. Anti-HEV IgG Avidity Testing: Utility for Diagnosing Acute and Resolved Genotype 3 Infections. Viruses 2021, 13, 236. https://doi.org/10.3390/ v13020236

Academic Editor: Helene Norder

Received: 12 January 2021

Accepted: 29 January 2021

Published: 3 February 2021

Publisher's Note: MDPI stays neutral with regard to jurisdictional claims in published maps and institutional affiliations.

Copyright: (c) 2021 by the authors. Licensee MDPI, Basel, Switzerland. This article is an open access article distributed under the terms and conditions of the Creative Commons Attribution (CC BY) license (https:// creativecommons.org/licenses/by/ $4.0 /)$.

\begin{abstract}
European Association of the Study of the Liver (EASL) guidelines specify HEV RNA, as well as anti-HEV IgG and IgM as positive markers for acute HEV infection. HEV RNA assay sensitivity limitations may lead to false negative test results in patients with low levels of viremia. Moreover, anti-HEV IgM positivity is not a reliable indicator for distinguishing between acute and resolved infections given the ability of this antibody to persist several months after a resolved infection. Our study aims were to assess HEV IgG avidity for diagnosing acute and resolved infections, regardless of the anti-HEV IgM serostatus, and examine assay reliability when evaluating different genotype 3 (GT3) HEV subtypes. Patient serum samples $(n=104)$ were tested for HEV IgG avidity by utilizing the DIA.PRO kit on a DSX automated instrument. Among patients identified with acute HEV infections, 32 were infected with GT3: GT3c $(n=5)$, GT3e $(n=8), 3 f(n=17)$ and GT3-unsubtyped $(n=2)$. Avidity sensitivity was $91.2 \%$ and specificity was $100 \%$. For patients with long-lasting antiHEV IgM persistence, an Avidity Index $>70 \%$ was observed. Thus, the DIA.PRO avidity assay may be utilized to distinguish between recently acquired and resolved HEV GT3 infections. However, for equivocal results (Avidity Index $>40-70 \%$ ), HEV RNA molecular testing will be required to confirm a recent infection.
\end{abstract}

Keywords: hepatitis E virus; IgG avidity test; IgM; HEV RNA; HEV genotype 3; HEV acute hepatitis

\section{Introduction}

Hepatitis E virus (HEV) is the main cause of acute viral hepatitis globally. It is estimated that there are approximately 20 million new infections every year with a worldwide yearly mortality rate of almost 70,000 people [1-3]. HEV is a single-stranded, positive sense RNA genome virus with a non-enveloped capsid, belonging to the genus Orthohepevirus of the Hepeviridae family. In general, human infections are linked to Orthohepevirus species A, which comprises eight genotypes [4]. Genotypes 1 and 2 are restricted to humans, while genotype 3 (GT3) and 4 (GT4) are considered zoonotic, causing cross-species infection in, for example, pigs, wild boars, deer, rabbits and rats. GT3 and GT4 are frequently transmitted to humans via contaminated food. HEV GT3 is predominantly found in Western countries [5] while GT4 mainly circulates in Asia [6], although some sporadic cases have also been identified in European countries [7,8]. Of the reported GT3 subtypes, GT3c, GT3e and GT3f have been isolated from European human samples [5,9-11]. GT7 and GT8 infect dromedary and Bactrian camels, respectively, with sporadic transmission to humans being reported for GT7 [12,13]. Recently, another HEV species, HEV-C (Orthohepevirus 
species C), which is generally isolated from rodents and ferrets, has also been detected in humans $[14,15]$.

Individuals with acute HEV infection can be symptomatic or asymptomatic. Reported symptoms are indistinguishable among the HEV genotypes and are very similar to those observed for hepatitis A virus acute infections. Symptoms can be non-specific (the prodromal or pre-icteric phase of an acute infection) and include asthenia, fever and pain. This may be followed by an icteric phase of morphologic changes (hepatocyte cytolysis) and biochemical changes in alanine amino transferase (ALT), aspartate aminotransferase (AST) or bilirubin [16]. Progression to a fulminant hepatitis has been observed in infected individuals with underlying liver disorders. Additionally, immunocompromised patients infected with HEV GT3 have developed chronic hepatitis [17].

A chronic infection is characterized by the persistence of HEV RNA viremia for more than six months after diagnosis [18]. While EASL guidelines indicate the detection of HEV RNA alone or with anti-HEV IgG and/or anti-HEV IgM as parameters for HEV acute infections [17], ECDC suggests that anti-HEV IgG plus IgM positivity is an indispensable indicator for HEV acute hepatitis. HEV RNA is the first indicator of infection and it remains detectable for approximately 10 to 52 days in serum [19]. However, when HEV RNA levels are $<100 \mathrm{IU} / \mathrm{mL}$, false negative test results are possible [19]. Moreover, the instability of naked HEV RNA may also influence assay sensitivity [20], in addition to sub-optimal storage and freeze/thaw techniques, as observed by conventional amplification methods (RT-PCR) [21] of other viral samples. Anti-HEV IgM can be detected within three to four days after the onset of jaundice [22] and persist up to 225 days after the onset of reported symptoms [23]. Detection of anti-HEV IgG is possible three to four days after anti-HEV IgM and persists for at least seven years. Anti-HEV IgG titre is variable and depends on the assay used for detection and thus, positivity is not useful for distinguishing between acute and resolved infections. IgG avidity is a quantitative serological test used to discriminate recent and resolved infections. IgG antibody binding power is weak in the early stage of infection, while it increases progressively in subsequent weeks due to affinity maturation and antigen-driven B cell selection. Avidity assays have been employed to differentiate acute or primary infections from persistent infections, recurrent infections, or the reactivated disease [24-26]. With respect to HEV infection, some studies have reported the role of anti-HEV IgG avidity in the improvement of diagnosis of acute infections $[27,28]$. However, this benefit requires further investigation in the context of different HEV subtypes and potential cross-reactivity. Our study aims to assess whether differentiation between acute and resolved HEV infections is possible by using the anti-HEV IgG avidity test, as well as to establish whether the reliability of the test is influenced by the GT3 subtype.

\section{Materials and Methods}

\subsection{Study Population}

Serum samples $(n=104)$ were collected from 101 patients and analysed. These samples were either obtained from patients recruited to the INMI L Spallanzani Hospital IRCCS or shipped to the virology lab for HEV RNA and antibody testing in a blinded fashion. This research was approved by the local Ethical Committee (n.70/2018). Sample data were stratified according to HEV disease status, serology and RT-PCR results. Group A ( $n=34)$ included sera from patients with a confirmed acute infection, as determined by positive results for HEV RNA and anti-HEV IgG and IgM. The HEV genotype and subtype status was evaluated for these patients. Group B $(n=16)$ included patients who had increases in transaminases, liver disorders, pain, or asthenia in the months prior to virological testing. At the time of sample collection, these patients had recovered from acute HEV infection, did not demonstrate chronic symptoms and exhibited decreases in transaminase levels. Testing indicated that these patients were HEV RNA negative and anti-HEV IgG and IgM positive (see Table 1$)$. Group C $(n=15)$ included patients who had been HEV RNA positive and recovering from acute infection. At the follow-up visit, collected samples were HEV RNA negative but positive for both anti-HEV IgG and IgM and liver function 
tests were now normal. These follow-up samples were collected 1 month to 2 years after the initial positive HEV RNA diagnosis (see Table 1). Group D included patients with resolved HEV infection $(n=5)$, as determined by anti-HEV IgG positive and IgM negative results. One patient (Pt71) was HEV RNA positive, although anti-HEV IgG and IgM were not detected. This patient was included in Group D to check the avidity test reliability in viremic HEV patients with acute infection, who has not already developed either anti-HEV IgG or anti-HEV IgM.

In order to investigate potential cross-reactivity of the DIA.PRO anti-HEV avidity assay, patient samples infected with hepatotropic viruses other than HEV were also evaluated. One patient (Pt72) was HEV RNA negative, anti-HEV IgG and IgM positive, HBV DNA positive and anti-HBc IgM positive. An acute hepatitis A (HAV) infection was confirmed in 12 patients (Pts 73-84) by HAV RNA RT-PCR testing, while an acute Epstein-Barr virus (EBV) infection was diagnosed in 10 patients (Pts 85-94) with the presence of EBV DNA and anti-virus capsid antigen (VCA) IgM in their blood. Ten patients (Pts 95-104) were CMV DNA positive and anti-cytomegalovirus (CMV) IgM positive. All these samples (Pts 73-104) were HEV RNA negative.

Detailed sample information is described in Table 1.

\subsection{Avidity Test}

The avidity test is based on the detection of dissociation of previously formed HEV IgG antigen immunocomplexes through a dissociation complex agent (urea) treatment. IgG antibodies bind weakly to the antigen during the initial stage of infection. After a month, this interaction strengthens and avidity increases progressively. Results are expressed as Avidity Index (AI), which is calculated as follows: $\mathrm{AI}=\mathrm{OD}_{450}$ (anti-HEV IgG in the urea-treated well) $/ \mathrm{OD}_{450}$ (anti-HEV IgG in the untreated well) $\times 100$ where $\mathrm{OD}_{450}$ is the optical density at a wavelength of 450 .

In this study, the avidity test was performed using the DIA.PRO HEV IgG assay, which is an enzyme-linked immunosorbent assay based on four synthetic peptides with conservative epitopes in ORF2 and ORF3 from genotypes 1, 2, 3, and 4 (DIA.PRO, Milan, Italy).

Serum samples were diluted in order to have an $\mathrm{OD}_{450}$ in the range of 0.460 to 3.3 . The assay was performed using the automated DSX Elisa processing system. Fresh tips were used to respectively transfer samples and reagents. This automated system allowed minimal hand-on time and yielded a result in $3 \mathrm{~h} 40 \mathrm{~min}$. The total assay volume was $200 \mu \mathrm{L}$ (pre-diluted 1:20). The liquid levels of samples, reagents, starter reagents, and system fluid were checked via sensors.

Duplicate samples were processed using DIA.PRO anti-HEV IgG enzyme-linked immunosorbent reagents.

One sample was prepared following standard procedures while the other was initially washed three times with $300 \mu \mathrm{L}$ urea (6M final concentration in washing buffer), which also included a $37^{\circ} \mathrm{C}$ soak for $5 \mathrm{~min}$ between washes. The assay was then performed according to the manufacturer's recommendations for both duplicates. The assay was repeated three times.

Results were categorized as follows: low Avidity Index, 1-40\%; equivocal results, $>40-70 \%$; high Avidity Index: $>70 \%$.

To confirm long-lasting anti-HEV IgM positivity, samples were evaluated using the "One site HEV IgM rapid test" (CTK Biotech, Poway, CA, USA). 
Table 1. Patient serological status, HEV RNA results and avidity index calculations.

\begin{tabular}{|c|c|c|c|c|c|c|c|c|c|}
\hline Sample Identification & Sex & Date of Sampling & HEV RT-PCR & HEV Genotype & $\begin{array}{c}\text { Mean Avidity } \\
\text { Index ( } \pm \text { SD) }\end{array}$ & HEV-IgG & HEV-IgM & Follow-Up $₫$ & Other \\
\hline \multicolumn{10}{|l|}{ Group A } \\
\hline Pt1 & $\mathrm{M}$ & $31 / 10 / 2019$ & + & $3 \mathrm{f}$ & $4.5(1.1)$ & + & + & & \\
\hline Pt2 & M & $27 / 01 / 2020$ & + & $3 \mathrm{f}$ & $9.0(1.7)$ & + & + & & \\
\hline $\mathrm{Pt3}$ & $\mathrm{F}$ & $13 / 08 / 2019$ & + & $3 e$ & $10.9(2.2)$ & + & + & & \\
\hline Pt4 & $\mathrm{F}$ & $30 / 09 / 2019$ & + & $3 e$ & $28.0(3.0)$ & + & + & & \\
\hline Pt5 & $\mathrm{M}$ & $18 / 10 / 2019$ & + & $3 f$ & 7.1 (3.4) & + & + & & \\
\hline Pt6 & $\mathrm{M}$ & $08 / 10 / 2019$ & + & $3 \mathrm{f}$ & $6.5(1.0)$ & + & + & & \\
\hline Pt7 & $\mathrm{M}$ & $04 / 11 / 2019$ & + & $3 \mathrm{f}$ & $14.9(1.5)$ & + & + & & \\
\hline $\mathrm{Pt} 8$ & $\mathrm{M}$ & $27 / 01 / 2020$ & + & $3 c$ & $20.7(3.8)$ & + & + & & \\
\hline Pt9 & M & $31 / 05 / 2017$ & + & 1 & $29.2(4.0)$ & + & + & & \\
\hline Pt10 & $\mathrm{M}$ & $06 / 12 / 2019$ & + & $3 \mathrm{f}$ & $9.0(1.6)$ & + & + & & \\
\hline Pt11 & $\mathrm{M}$ & $22 / 07 / 2019$ & + & $3 e$ & $4.4(1.1)$ & + & + & & \\
\hline Pt12 & $\mathrm{M}$ & $27 / 08 / 2019$ & + & $3 e$ & $5.5(0.6)$ & + & + & & \\
\hline Pt13 & $\mathrm{M}$ & $10 / 09 / 2019$ & + & $3 e$ & $7.9(0.3)$ & + & + & & \\
\hline Pt14 & $\mathrm{M}$ & $26 / 01 / 2018$ & + & $3 c$ & $4.8(0.7)$ & + & + & & \\
\hline Pt15 & $\mathrm{M}$ & $17 / 03 / 2018$ & + & $3 \mathrm{f}$ & 14.9 (12.4) & + & + & & \\
\hline Pt16 & $\mathrm{M}$ & $08 / 05 / 2020$ & + & $3 c$ & $7.7(1.0)$ & + & + & & \\
\hline Pt17 & $\mathrm{M}$ & $17 / 01 / 2019$ & + & $3 c$ & $6.3(2.5)$ & + & + & & \\
\hline Pt18 & $\mathrm{M}$ & $18 / 01 / 2019$ & + & $3 \mathrm{f}$ & $19.6(2.6)$ & + & + & & \\
\hline Pt19 & $\mathrm{M}$ & $24 / 08 / 2019$ & + & $3 e$ & 20.2 (3.6) & + & + & & \\
\hline Pt21 & $\mathrm{M}$ & $03 / 12 / 2019$ & + & 3 & $25.3(1.3)$ & + & + & & \\
\hline Pt22 & $\mathrm{M}$ & $21 / 01 / 2016$ & + & $3 c$ & $22.3(0.8)$ & + & + & & \\
\hline Pt23 & $\mathrm{M}$ & $03 / 03 / 2018$ & + & $3 \mathrm{f}$ & $28.3(0.8)$ & + & + & & \\
\hline Pt24 & $\mathrm{M}$ & $27 / 06 / 2020$ & + & $3 f$ & $37.1(2.4)$ & + & + & & \\
\hline Pt25 & $\mathrm{M}$ & $03 / 02 / 2016$ & + & $3 \mathrm{f}$ & $16.3(0.5)$ & + & + & & \\
\hline Pt26 & $\mathrm{M}$ & $24 / 08 / 2019$ & + & $3 e$ & $2.9(0,3)$ & + & + & & \\
\hline Pt27 & $\mathrm{M}$ & $25 / 05 / 2017$ & + & $3 \mathrm{f}$ & $35.5(1.2)$ & + & + & & \\
\hline Pt28 & $\mathrm{M}$ & $01 / 03 / 2016$ & + & $3 e$ & $54.1(6.2)$ & + & + & & \\
\hline Pt29 & $\mathrm{M}$ & $30 / 03 / 2018$ & + & $3 f$ & $8.0(1.0)$ & + & + & & \\
\hline Pt30 & $\mathrm{F}$ & $05 / 05 / 2018$ & + & $3 \mathrm{f}$ & $5.1(0.6)$ & + & + & & \\
\hline Pt31 & $\mathrm{M}$ & $31 / 05 / 2018$ & + & $3 f$ & $11.9(0.4)$ & + & + & & \\
\hline Pt32 & $\mathrm{M}$ & $14 / 07 / 2018$ & + & $3 \mathrm{f}$ & $6.0(0.2)$ & + & + & & \\
\hline Pt33 & $\mathrm{M}$ & $13 / 06 / 2019$ & + & 1 & $44.5(0.7)$ & + & + & & \\
\hline Pt34 & $\mathrm{F}$ & $23 / 06 / 2020$ & + & 3 & $63.4(1.2)$ & + & + & & \\
\hline \multicolumn{10}{|l|}{ Group B } \\
\hline Pt35 & $\mathrm{M}$ & $17 / 10 / 2018$ & - & n.d. & $83.7(0.8)$ & + & + & & \\
\hline Pt36 & $\mathrm{M}$ & $16 / 11 / 2019$ & - & n.d. & $95.0(0.9)$ & + & + & & \\
\hline Pt37 & $\mathrm{F}$ & $17 / 12 / 2019$ & - & n.d. & $71.2(2.7)$ & + & + & & \\
\hline Pt38 & $\mathrm{M}$ & $31 / 08 / 2019$ & - & n.d. & $49.1(1.3)$ & + & + & & \\
\hline Pt39 & $\mathrm{F}$ & $25 / 10 / 2019$ & - & n.d. & $18.1(0.3)$ & + & + & & \\
\hline
\end{tabular}


Table 1. Cont.

\begin{tabular}{|c|c|c|c|c|c|c|c|c|c|}
\hline Sample Identification & Sex & Date of Sampling & HEV RT-PCR & HEV Genotype & $\begin{array}{l}\text { Mean Avidity } \\
\text { Index ( } \pm \text { SD) }\end{array}$ & HEV-IgG & HEV-IgM & Follow-Up $\S$ & Other \\
\hline Pt 40 & $\mathrm{~F}$ & $26 / 10 / 2019$ & - & n.d. & $59.2(0.4)$ & + & + & & \\
\hline Pt41 & $\mathrm{M}$ & $28 / 03 / 2018$ & - & n.d. & $87.6(0.9)$ & + & + & & \\
\hline Pt42 & M & $07 / 03 / 2018$ & - & n.d. & $79.8(0.4)$ & + & + & & \\
\hline Pt43 & $\mathrm{M}$ & $19 / 06 / 2019$ & - & n.d. & $92.5(0.7)$ & + & + & & \\
\hline Pt44 & M & $30 / 08 / 2019$ & - & n.d. & $100.0(0.5)$ & + & + & & \\
\hline Pt45 & $\mathrm{F}$ & $21 / 01 / 2019$ & - & n.d. & $100.0(0.0)$ & + & + & & \\
\hline Pt46 & $\mathrm{M}$ & $22 / 01 / 2019$ & - & n.d. & 38.8 (1.1) & + & + & & \\
\hline Pt47 & $\mathrm{F}$ & $26 / 10 / 2019$ & - & n.d. & $64.6(1.0)$ & + & + & & \\
\hline Pt48 & $\mathrm{M}$ & $30 / 10 / 2019$ & - & n.d. & $34.7(20.1)$ & + & + & & \\
\hline Pt49 & $\mathrm{M}$ & $19 / 10 / 2016$ & - & n.d. & $100.0(0.0)$ & + & + & & \\
\hline Pt50 & M & $13 / 11 / 2019$ & - & n.d. & $100.0(0.0)$ & + & + & & \\
\hline \multicolumn{10}{|l|}{ Group C } \\
\hline Pt51 & $\mathrm{M}$ & $20 / 02 / 2016$ & - & n.d. & $98.4(0.4)$ & + & + & 8 months & \\
\hline Pt52* & M & $22 / 02 / 2020$ & - & n.d. & $100.0(0.0)$ & + & + & 5 months & \\
\hline Pt53 *** & $\mathrm{M}$ & $12 / 02 / 2018$ & - & n.d. & $98.9(0.3)$ & + & + & 24 months & \\
\hline Pt54 & M & $30 / 08 / 2019$ & - & n.d. & 99.4 (1.1) & + & + & 14 months & \\
\hline Pt55 & $\mathrm{M}$ & $14 / 10 / 2018$ & - & n.d. & $100.0(0.0)$ & + & + & 7 months & \\
\hline Pt56 & $\mathrm{M}$ & $23 / 04 / 2018$ & - & n.d. & $100.0(0.0)$ & + & + & 7 months & \\
\hline Pt57 & $\mathrm{F}$ & $28 / 10 / 2018$ & - & n.d. & $99.8(0.2)$ & + & + & 14 months & \\
\hline Pt60 *** & $\mathrm{M}$ & $19 / 05 / 2018$ & - & n.d. & $99.2(0.7)$ & + & + & 27 months & \\
\hline Pt61 & $\mathrm{M}$ & $23 / 03 / 2020$ & - & n.d. & $100.0(0.0)$ & + & + & 24 months & \\
\hline Pt62 & $\mathrm{M}$ & $15 / 03 / 2018$ & - & n.d. & $96.3(0.8)$ & + & + & 12 months & \\
\hline Pt63 ** & $\mathrm{F}$ & $04 / 12 / 2019$ & - & n.d. & $79.5(1.3)$ & + & + & 4 months & \\
\hline Pt64 & $\mathrm{M}$ & $16 / 02 / 2016$ & - & n.d. & 28.5 & + & + & 1 month & \\
\hline Pt65 & $\mathrm{M}$ & $19 / 07 / 2018$ & - & n.d. & $95.8(1.4)$ & + & + & 25 months & \\
\hline \multicolumn{10}{|l|}{ Group D } \\
\hline Pt66 & $\mathrm{M}$ & $23 / 10 / 2016$ & - & n.d. & $100.0(0.0)$ & + & - & & \\
\hline Pt67 & $\mathrm{F}$ & $24 / 11 / 2015$ & - & n.d. & $100.0(0.0)$ & + & - & & \\
\hline Pt68 & $\mathrm{M}$ & $05 / 12 / 2015$ & - & n.d. & $72.2(5.6)$ & + & - & & \\
\hline Pt69 & $\mathrm{M}$ & $11 / 05 / 2020$ & - & n.d. & $93.8(2.5)$ & + & - & & \\
\hline Pt70 & $\mathrm{M}$ & $11 / 05 / 2020$ & - & n.d. & $98.6(0.6)$ & + & - & & \\
\hline Pt71 & $\mathrm{F}$ & $17 / 06 / 2020$ & + & $3 c$ & n.d. & - & - & & \\
\hline Pt72 & M & $03 / 06 / 2019$ & - & n.d. & $100.0(0.0)$ & + & + & & anti_HBc IgM +, HBV DNA + \\
\hline Pt73-Pt84 & $\mathrm{M}=11 / \mathrm{F}=1$ & $02 / 03 / 2017-15 / 04 / 2020$ & - & n.d. & n.d. & - & - & & HAV RNA + \\
\hline Pt85-Pt94 & $\mathrm{M}=6 / \mathrm{F}=4$ & $23 / 02 / 2016-26 / 09 / 2020$ & - & n.d. & n.d. & - & - & & VCA_IgM +, EBV DNA + \\
\hline Pt95-Pt104 & $\mathrm{M}=0 / \mathrm{F}=10$ & $05 / 06 / 2020-11 / 06 / 2020$ & - & n.d. & n.d. & - & - & & anti_CMV-IgM, CMV DNA + \\
\hline
\end{tabular}




\subsection{Data Analysis}

Assay sensitivity was calculated for Group A samples as follows: true positive/(true positive + false negative) $\times 100$ ). Assay specificity was calculated in Group D (Pts73-104) samples as follows: true negative/(true negative + false positive) $\times 100$.

The comparison of AI in different GT3 subtypes was performed using the MannWhitney test with GraphPad Prism version 8.0.2 (GraphPad software, San Diego Inc., CA, USA). A $p$-value $<0.05$ was considered statistically significant.

The Wald test was employed to calculate the kappa value and was performed using the Analyse-it software, version 5.65.3. A kappa value $>0.70$ indicated high agreement, 0.40 to 0.70 indicated moderate (or good) agreement and $<0.40$ indicated poor agreement.

\subsection{Phylogenetic Analysis}

HEV RNA was isolated from $400 \mu \mathrm{L}$ of serum using the QIASYMPHONY automated instrument (QIAGEN, Hilden, Germany). All samples collected before 2019 were retrotranscribed employing the QIAGEN Onestep RT-PCR kit with primers for the HEV ORF2 region as previously described [29]. For samples obtained since 2019, RT-PCR was performed by Super Script IV PCR with random primers (SSIV) (Thermofisher Scientific, Paisley, UK), according to the manufacturer's instructions. Amplification of cDNA was achieved by a nested polymerase chain reaction protocol (PCR) using TaqGold polymerase and two sets of primers encompassing a $457 \mathrm{bp}$ fragment within the ORF2 gene, as used in the OneStep protocol [29]. Cycling conditions for first and second round PCR were as follows: (1) $95{ }^{\circ} \mathrm{C}$ (15 min), (2) $94{ }^{\circ} \mathrm{C}(30 \mathrm{~s})$, (3) $56^{\circ} \mathrm{C}$ (30 s) and (4) $72{ }^{\circ} \mathrm{C}$ (45 s); steps $2-4$ were repeated 35 times. A final extension was performed at $72^{\circ} \mathrm{C}$ for $10 \mathrm{~min}$. ORF2 RT-PCR negative samples were retested with primers against a conserved ORF1 region [30]. RNA was retro-transcribed in the presence of random hexamers and SSIV. cDNA $(10 \mu \mathrm{L})$ was used as template in the first round PCR with outer primers 1679 and 1680 while nested primers 1681 and 1682 were employed for second-round PCR [30]. Cycling conditions for PCR were: $94{ }^{\circ} \mathrm{C}, 15 \mathrm{~min} ; 40$ cycles at $94{ }^{\circ} \mathrm{C}$ for $30 \mathrm{~s}, 50{ }^{\circ} \mathrm{C}$ for $45 \mathrm{~s}$ and $72{ }^{\circ} \mathrm{C}$ for $1 \mathrm{~min}$; and a final extension at $72{ }^{\circ} \mathrm{C}$ for $7 \mathrm{~min}$.

Direct sequencing of both PCR product strands using second round amplification primers was achieved on the ABI PRISM 3100 automated sequences (Applied Biosystems, Forster, CA, USA). Following a preliminary BLAST analysis, the genotype and subtype of each isolate was confirmed by phylogenetic analysis. Sequences were aligned using the MEGA X software package [31] and the maximum-likelihood method based on General Time Reversible (GTR) model $+\mathrm{G}+\mathrm{I}$ with 500 bootstrap replicates including reference sequences [4]. The phylogenetic tree was visualized with FigTree v. 1.4.4.

Two strains were not included in the phylogenetic analysis since Sanger sequencing only led to resolution of short fragments; these strains were classified as GT3 unsubtyped (Pt21 and Pt34).

\section{Results}

The calculated AI was employed to differentiate samples from patients with recent versus resolved HEV infections. Qualitative AIs are listed in Table 1. In group A $(n=34)$, which included samples positive for HEV RNA and anti-HEV IgG/IgM, two were segregated with GT1 while the majority $(n=32)$ were segregated with GT3. The GT3 sequences clustered with the following subtypes or clades: GT3c $(n=5)$, GT3e $(n=8)$, GT3f $(n=17)$ (Figures 1 and 2) while two were GT3-unsubtyped. The majority of group A samples $(n=31)$ had a low $\mathrm{AI}(<40 \%)$; the mean $\mathrm{AI}$ was $17.5 \%$. 


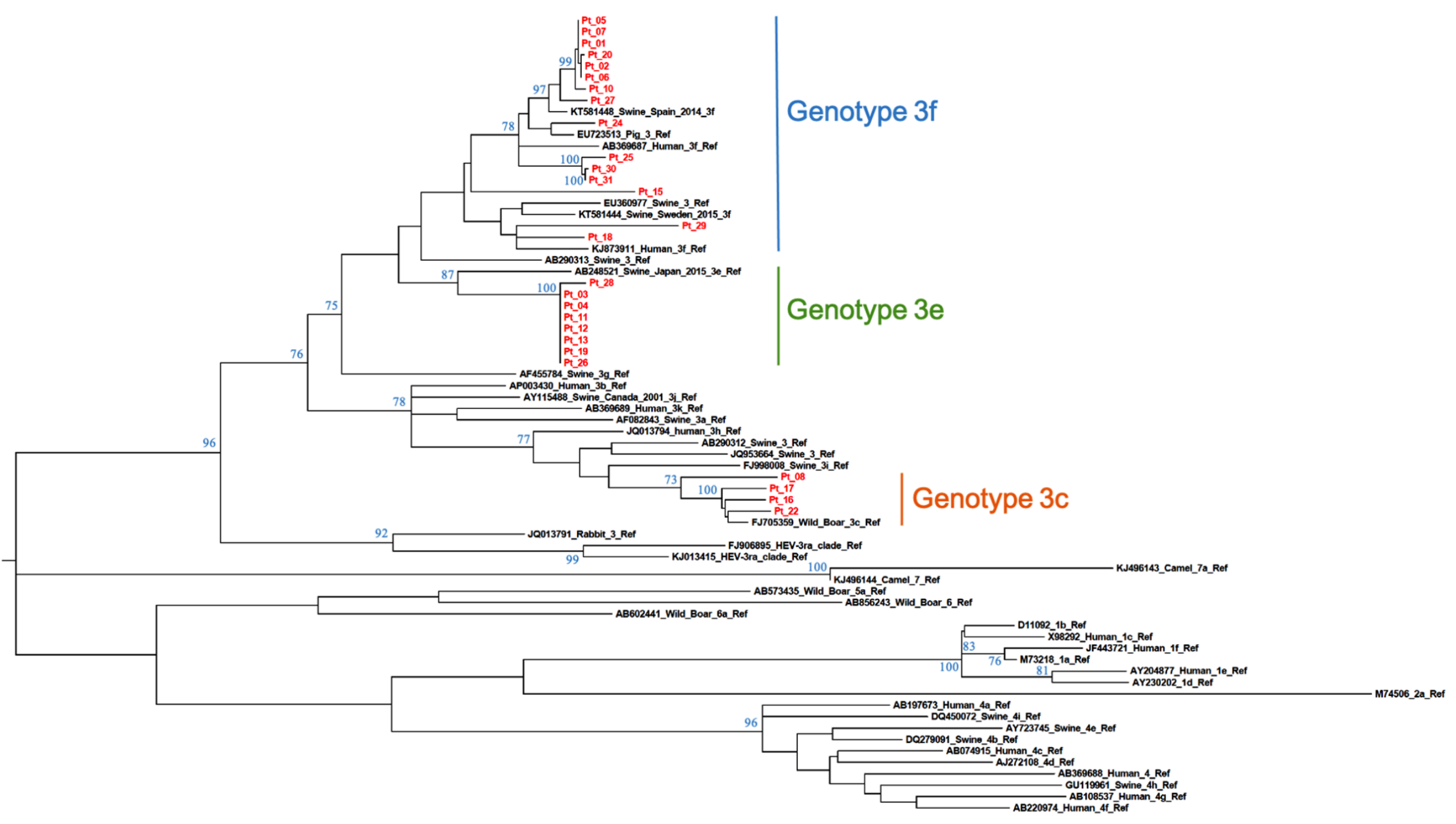

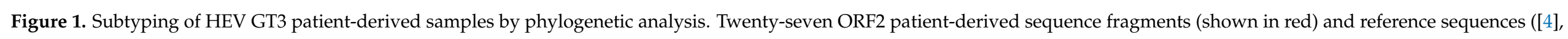

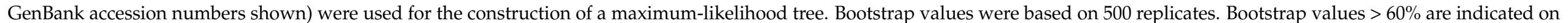

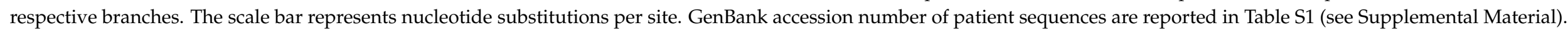




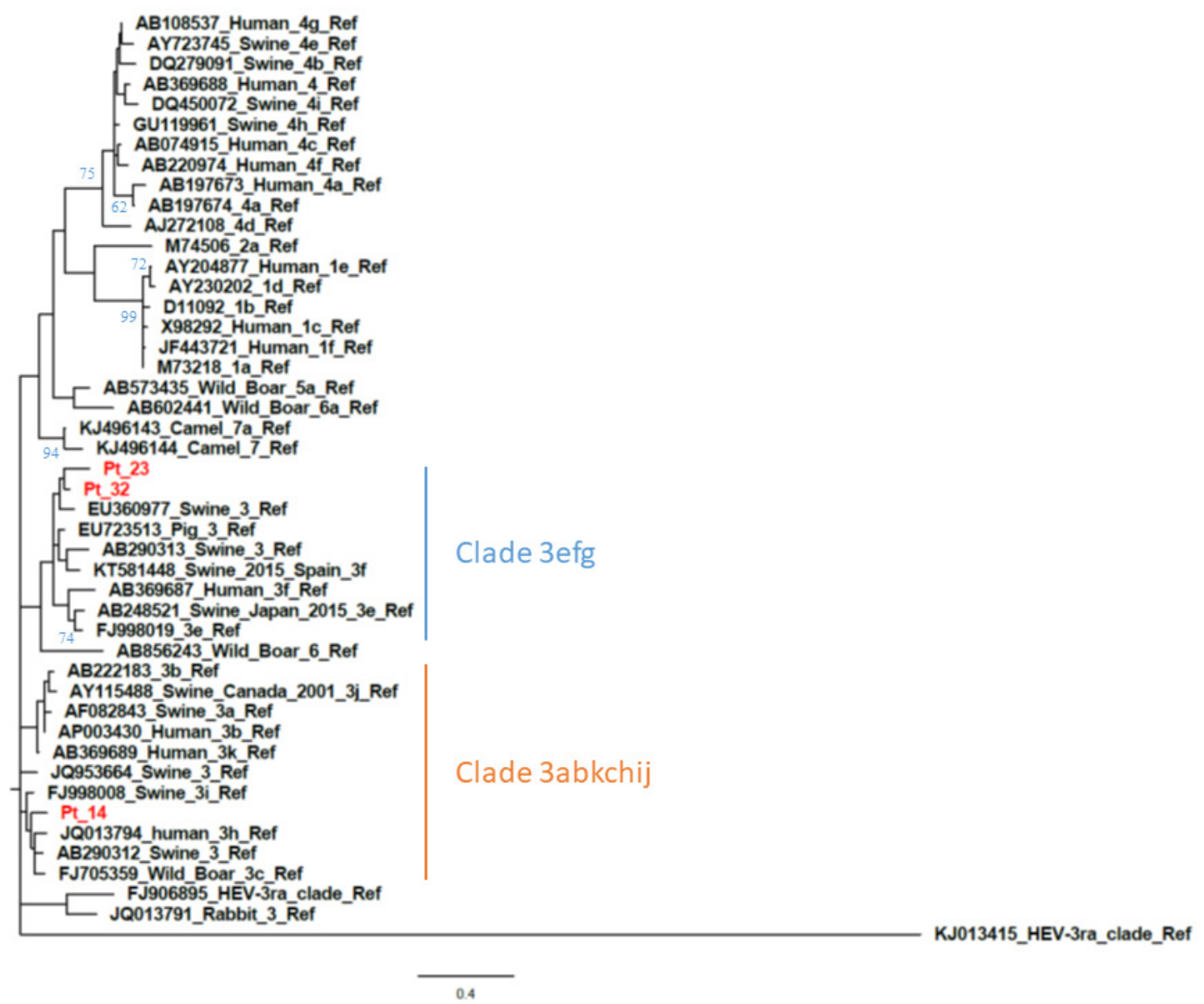

Figure 2. Phylogenetic analysis based on partial open reading frame 1 (ORF1) (125 nt, nt78-202 of the HE-JA04-1911 isolate, GenBank accession number AB248521) sequences from patient-derived samples with a negative ORF2 RT-PCR result. The phylogenetic tree includes the prototype strains as indicated by Smith et al. [4] The maximum-likelihood tree was constructed with a bootstrap of 500 replicates. Bootstrap values $>60 \%$ are indicated on respective branches. The scale bar represents nucleotide substitutions per site. Supplementary data on sequence accession number of patients are provided in Table S1 (see Supplemental Material).

Similar AI values were observed irrespective of GT3 subtype: mean AIs for GT3c, GT3e and GT3f were $12.4 \pm 8.4,16.8 \pm 17.4$, and $14.1 \pm 10.4$, respectively. Therefore, in this limited sample set, AI values were not influenced by GT3 subtype $(p>0.05)$ (Figure 3$)$.

For the three patient-derived samples with an $\mathrm{AI}>40 \%$, one (Pt33) was a Bengali migrant who had an AI of $44.5 \%$ and was hospitalized for a confirmed GT1 acute infection after returning from Bangladesh. Since GT1 is endemic in Bangladesh, it is possible that this equivocal AI value was related to the patient being reinfected; therefore, anti-HEV IgG binding to antigen could be stronger even in the early phase of reinfection. Pt34 $(\mathrm{AI}=64.7 \%)$ was tested for the presence of anti-HEV IgG and IgM after confirming the absence of other types of viral hepatitis (HAV, HBV and HCV). This patient was still in the late phase of acute infection with detectable HEV RNA, and exhibited a high anti-HEV IgM index (11.5 S/CO) and an O.D. of 3.140, suggesting seroconversion 1-3 months earlier. On subsequent testing a month later, Pt34 was HEV RNA negative. Pt28 (AI $=51.2 \%)$ was first shown to be HEV RNA positive two months earlier; this patient was receiving antiviral treatment (sofosbuvir plus ribavirin) for HEV infection. 


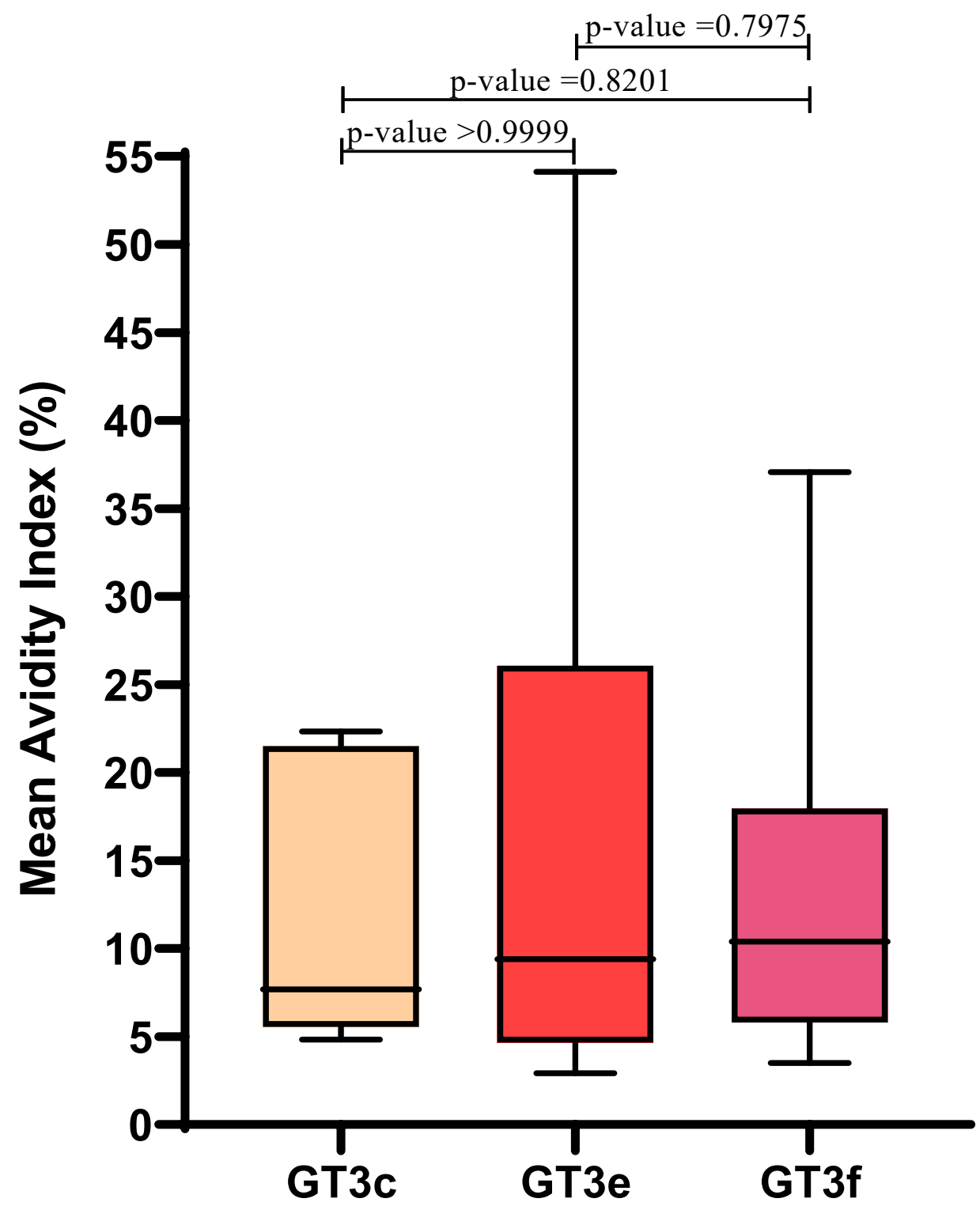

Figure 3. Correlation between HEV GT3 subtypes (GT3c, $n=5$; GT3e, $n=8$; GT3f, $n=17$ ) and the mean Avidity Index, which was calculated for Group A GT3 patients. Boxes represent the first and third quartiles and the black line represents the median value. $p$-values were calculated using the Mann-Whitney test.

In Group B $(n=16)$, the mean $\mathrm{AI}$ was $73.5 \% \pm 26.8 \%$, and there were three patients with low $\mathrm{AI}(<40 \%)$. One female patient (Pt39) with an AI of $18.6 \%$ was pregnant and anti-HEV IgG/IgM positive with transaminase elevation, nausea and pain. All other hepatitis markers were negative, including anti-Toxoplasma gondii IgG and IgM. The other two patients (Pt46 and Pt48) had acute hepatitis clinical manifestations although negative for HEV RNA as well as for cytomegalovirus (CMV), EBV, HAV and HBV serological markers. Pt46 (AI $=39.1 \%)$ was hospitalized in a different medical centre. The shipped serum sample had not been stored at an optimal temperature; therefore, it is possible that HEV RNA degradation may have occurred before testing. Pt48 ( $\mathrm{AI}=35.5 \%)$ had clinical symptoms indicative of acute infections 6-8 weeks before HEV RNA and antibody testing.

Equivocal values for anti-HEV IgG AI were observed for three patients (Pt38, Pt40 and Pt47) who recovered from hepatitis: they had normalization of their ALT, AST, and total bilirubin values. Speculation that HEV serological testing was performed in the late 
stages of acute infection could not be confirmed since the timing for onset of symptoms was unknown.

Group $C$ represented single or double time-point convalescent patient-derived samples collected 1-27 months after a HEV RNA positive result. In this group, the mean AI was $90.2 \% \pm 20.9 \%$. Three patients were sampled sequentially: two were first sampled during the early stage of acute infection when HEV RNA was positive (Pt3 and Pt12 from Group A) and then approximately $4-5$ months later; the third patient ( $\mathrm{Pt53}$ ) was sampled twice during follow-up (Table 1). All Group C patients with persistent ( $>6$ months) antiHEV IgM exhibited an AI $>70 \%$ (Table 2). Patients with a follow-up period $>3$ months also showed an AI value $>70 \%$. It is feasible that Pt64 (AI $=28.6 \%)$, who was HEV RNA positive one month before the AI determination, were still experiencing a late phase acute infection. Overall, the results demonstrated that low values of AI were indicative of a recent HEV infection, whereas high values of AI suggested a resolved infection. Samples from four patients with persistent anti-HEV IgM for $\geq 24$ months were also tested with the "One site HEV IgM rapid test". All samples were anti-HEV IgM positive (Figure 4). This further confirmed long-lasting IgM positivity.

Table 2. Group C HEV IgG avidity results categorized by the estimated time since HEV RNA detection.

\begin{tabular}{cccc}
\hline Time of AI Detection & Low AI & Equivocal AI & High AI \\
\hline$\geq 5$ months & 0 & 0 & 11 \\
$>2$ month, $<5$ months & 0 & 0 & 2 \\
$>1$ month, $\leq 2$ months & 0 & 1 & 0 \\
$\leq 1$ month & 1 & 0 & 0
\end{tabular}

AI, Avidity Index; Low AI: 1-40\%; Equivocal AI: >40-70\%; High AI: >70\%.

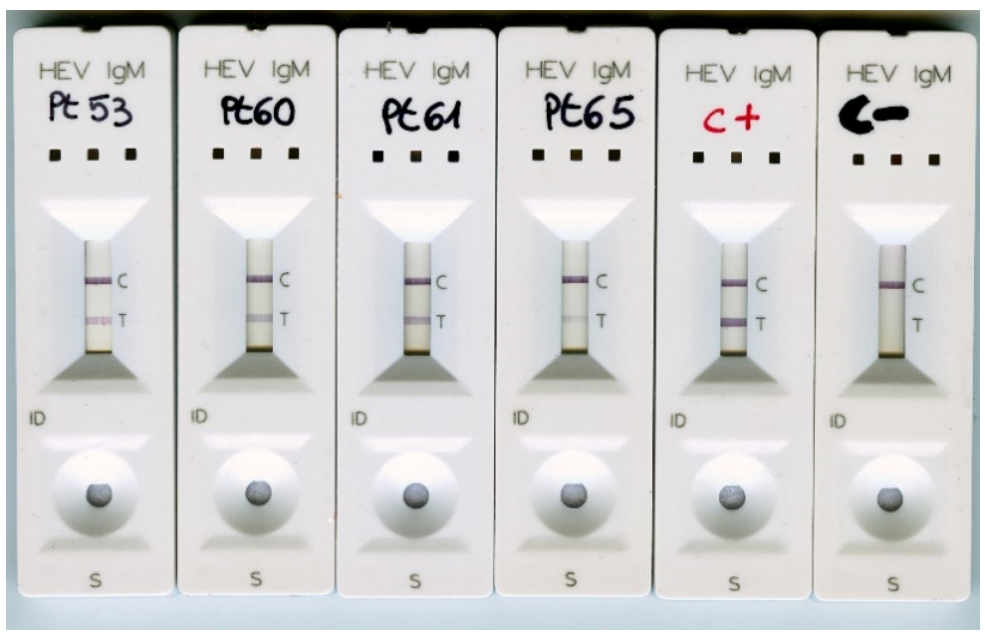

Figure 4. Group C patient samples with persistent ( $>24$ months) anti-HEV IgM positivity were tested using the anti-HEV IgM rapid test. $\mathrm{C}+$, positive control (HEV RNA positive, anti-HEV IgM positive, acute infection); C-, negative control (anti-HEV IgG positive, anti-HEV IgM negative, resolved HEV infection).

Group D patient samples were selected to assess both cross-reactivity and specificity of the AI assay. All HAV-positive samples $(n=12)$, HBV-positive samples $(n=1)$, EBV antiVCA IgM positive samples $(n=10)$, and anti-CMV IgM positive samples $(n=10)$ produced negative HEV IgG avidity results. Five samples from patients who were anti-HEV IgG positive and IgM negative yielded an AI $>70 \%$. The DIA.PRO HEV IgG avidity test showed no cross-reactivity (samples from Pts 66-104). One patient (Pt71) was viremic and infected with a GT3c strain and, as expected for an early stage acute infection, was negative for both anti-HEV IgG and IgM and had an indeterminate AI. Data analyses indicated that the AI assay sensitivity was $91.2 \%$, the specificity was $100 \%$, and the accuracy was $90.5 \%$. 
When HEV RNA positive patients and patients with clinical manifestations associated with acute infection (P28, Pt38, Pt40, Pt85) were considered as having a recent infection, the agreement between a low $\mathrm{AI}$ and recent infection was $97.4 \%$, while the kappa value, as determined by the Wald Test, was 92.3 (Figure 5).

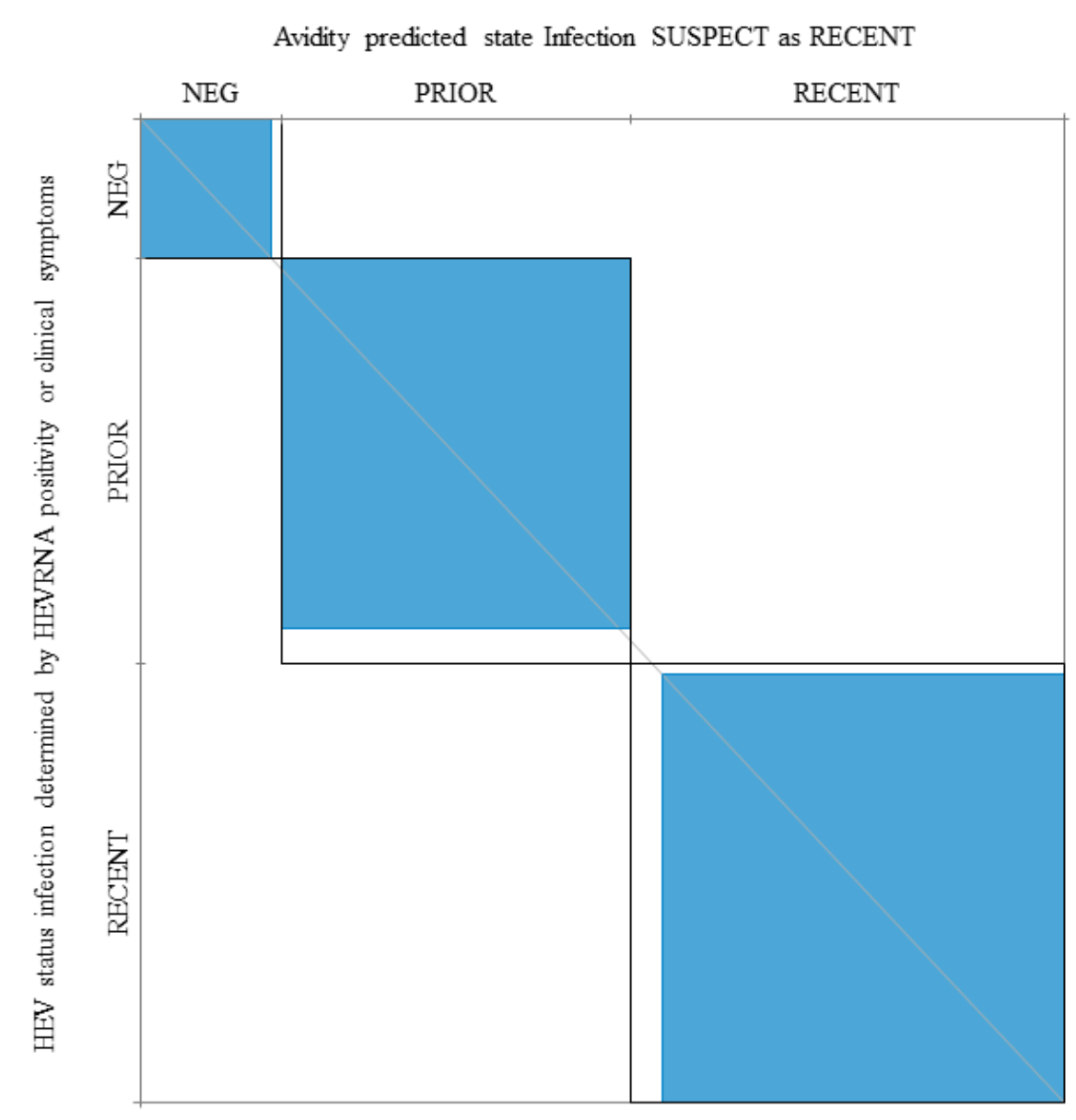

Figure 5. Power of the IgG avidity test in prediction of recent and prior (resolved) infections when considering HEV RNA positivity and clinical manifestations. Neg, patients who are HEV RNA negative and anti-HEV IgM negative. The kappa statistic was employed to measure the agreement between the Avidity Index (AI), HEV RNA, serological markers and clinical symptoms (see Section 3).

\section{Discussion}

Serological tests such as those detecting anti-HEV IgG and IgM, and molecular tests measuring HEV RNA, are considered the basis for the diagnosis and differentiation between acute and resolved hepatitis E infections [17]. In clinical practice, however, anti-HEV IgM positivity in HEV RNA negative individuals does not provide any clear indication of the infection status, especially since anti-HEV IgM antibodies may persist for months or even years [22,32] following the acute phase of infection. A greater understanding regarding the utility of avidity values for diagnosing HEV disease stage in patients with long-lasting anti-HEV IgM positivity, as well as HEV GT3 subtype effects on avidity test performance is required.

In our study, the performance of the commercially available DIA.PRO assay was evaluated. This assay was employed as an anti-HEV IgG diagnostic test for measuring IgG avidity in HEV RNA positive patients primarily infected with GT3. These GT3 strains were subtyped to assess the impact of subtype on assay proficiency. For Group A patients who were viremic and positive for anti-HEV IgM, the avidity assay sensitivity was $91.2 \%$, 
confirming results previously described by other authors [27,28]. Assay specificity was $100 \%$, indicating that pathogens such as HAV, which causes acute hepatitis with symptoms similar to those of hepatitis $\mathrm{E}$, were not cross-reactive with the HEV avidity determination. In addition to $\mathrm{HAV}, \mathrm{HBV}, \mathrm{CMV}$ and $\mathrm{EBV}$, active infections did not appear to interfere with the HEV avidity determination; however, further analyses are recommended to confirm these findings.

Only three $(<10 \%)$ viremic patients (Pt28, Pt33, Pt34) with acute infection showed equivocal AI results.

In contrast to the Bigaillon study [27], we observed no false low-avidity values in sera from immunocompetent patients with a follow-up period $>4$ months after HEV RNA detection.

However, the relatively low number of samples did not allow any conclusive remarks.

Even Pt63, who was anti-HEV IgM positive four months after being HEV RNA positive, had a high-avidity value (mean $\mathrm{AI}=79.5 \%$ ). Therefore, in our study, a low IgG AI, measured using the DIA.PRO assay, appeared to predict an infection in the early stages (first 3-4 months), while a high IgG AI excluded patients with a primary infection occurring for $<4$ months. AI results within the equivocal range $(>40-70 \%)$ are more difficult to interpret. As shown in Table 1, three Group A viremic patients (Pt28, Pt33 and Pt34), diagnosed by clinicians as having an acute HEV infection, and three Group B patients (Pt38, Pt40 and Pt47), with ALT normalization at the time of AI determination, had AIs in the $>40-70 \%$ range. Additionally, a Group C patient (Pt58) had an equivocal AI two months after being HEV RNA positive. Some equivocal results may be linked to reinfection (Pt33) or a difference in maturation time for IgG avidity (Pt34); however, limitations of available anamnestic data and follow-up serum samples prevented us from confirming these assertions.

No statistically significant differences in AI sensitivity were observed between Group A GT3 viremic patients, irrespective of GT3 subtype (Figure 3). This is notable as it reinforces the value of the avidity test for assessment of acute HEV infections. Recently, Riviero-Barciela proposed this using HEV antigen (HEV-Ag) as a serological marker to discriminate between recent and resolved HEV infections in patients positive for anti-HEV IgM. The authors calculated a predictive positive value of $100 \%$ and a negative predictive value of $44 \%$ for the HEV-Ag test, resulting in a diagnostic accuracy of 57\% [32], and suggested using this test to exclude primary infection in patients with persistent anti-HEV IgM. Of note, HEV-Ag was shown to be weakly sensitive in asymptomatic blood donors [33] and in patients with HEV acute infection [34] where HEV-Ag sensitivity was reported as $40 \%$ and $46.7 \%$, respectively. Additionally, Zhao demonstrated that the presence of high IgG concentrations reduced the sensitivity of their HEV-Ag assay [35]. Moreover, Trémaux demonstrated that HEV-Ag assay sensitivity varied depending on GT3 subtype [36]; the lower limit of detection differed by one or two logarithmic units when comparing GT3c versus GT3f. Conversely, our results suggested that AI was not affected by the GT3 subtype during the assessment of acute infections and we were able to identify recent infections in HEV RNA-negative patients with clinical symptoms.

\section{Conclusions}

Results described in this study support the application of the anti-HEV IgG avidity ELISA test for distinguishing between recently acquired and resolved infections, irrespective of anti-HEV IgM positivity status. Although the sample size was limited, the results suggested that the avidity test performance appeared to be reliable for acute infection assessments of HEV GT3 subtypes, which is in contrast to observations with the reported HEV-Ag assay [36]. For avidity tests yielding equivocal AI values, potentially caused by the variability in timing for IgG maturation, recent HEV infections should be confirmed by HEV RNA detection. 
Supplementary Materials: The following are available online at https:/ / www.mdpi.com/1999-491 5/13/2/236/s1, Table S1: GenBank accession numbers for sequences included in phylogenetic trees targeting ORF1 or ORF2.

Author Contributions: A.R.G. designed the study, analysed the data and drafted the manuscript. A.C. performed the serological test. C.M. analysed the data and performed the phylogenetic analysis. G.D., C.T., R.L. collected clinical information and analysed the data. D.L. collected samples and analysed the data. F.R. set up the avidity protocol. F.M. reviewed and revised the manuscript. M.R.C. found resource and revised the manuscript. All authors have read and agreed to the published version of the manuscript.

Funding: This work was supported by the Ricerca Corrente Funding scheme from the Italian Minister of Health.

Institutional Review Board Statement: The study was conducted according to the guidelines of the Declaration of Helsinki, and approved by the Institutional Review Board of INMI L Spallazani IRCCS (protocol code 70/2018, date of approval: 3 January 2019).

Informed Consent Statement: Patient consent was waived because the study has not any impact on the clinical management of patients and in their health status.

Acknowledgments: We would like to thank Gianluca Rinaldi for technical assistance and Mariana Badescu for editing.

Conflicts of Interest: The authors declare no conflict of interest.

\section{References}

1. Rein, D.B.; Stevens, G.A.; Theaker, J.; Wittenborn, J.S.; Wiersma, S.T. The global burden of hepatitis E virus genotypes 1 and 2 in 2005. Hepatology 2012, 55, 988-997. [CrossRef] [PubMed]

2. GBD 2013 Mortality and Causes of Death Collaborators. Global, regional, and national age-sex specific all-cause and cause-specific mortality for 240 causes of death, 1990-2013: A systematic analysis for the Global Burden of Disease Study 2013. Lancet 2015, 385, 117-171. [CrossRef]

3. Debing, Y.; Moradpour, D.; Neyts, J.; Gouttenoire, J. Update on hepatitis E virology: Implications for clinical practice. J. Hepatol. 2016, 65, 200-212. [CrossRef]

4. Smith, D.B.; Izopet, J.; Nicot, F.; Simmonds, P.; Jameel, S.; Meng, X.J.; Norder, H.; Okamoto, H.; van der Poel, W.H.M.; Reuter, G.; et al. Update: Proposed reference sequences for subtypes of hepatitis E virus (species Orthohepevirus A). J. Gen. Virol. 2020, 101, 692-698. [CrossRef]

5. Izopet, J.; Tremeaux, P.; Marion, O.; Migueres, M.; Capelli, N.; Chapuy-Regaud, S.; Mansuy, J.M.; Abravanel, F.; Kamar, N.; Lhomme, S. Hepatitis E virus infections in Europe. J. Clin. Virol. 2019, 120, 20-26. [CrossRef]

6. Nakano, T.; Takahashi, K.; Takahashi, M.; Nishigaki, Y.; Watanabe, N.; Ishida, S.; Fujimoto, S.; Kato, H.; Okano, H.; Takei, Y.; et al. Investigating the origin and global dispersal history of hepatitis E virus genotype 4 using phylogeographical analysis. Liver Int. 2016, 36, 31-41, Epub 26 June 2015. [CrossRef] [PubMed]

7. Garbuglia, A.R.; Scognamiglio, P.; Petrosillo, N.; Mastroianni, C.M.; Sordillo, P.; Gentile, D.; La Scala, P.; Girardi, E.; Capobianchi, M.R. Hepatitis E virus genotype 4 outbreak, Italy, 2011. Emerg. Infect. Dis. 2013, 19, 110-114. [CrossRef]

8. Bouamra, Y.; Gérolami, R.; Arzouni, J.P.; Grimaud, J.C.; Lafforgue, P.; Nelli, M.; Tivoli, N.; Ferretti, A.; Motte, A.; Colson, P. Emergence of autochthonous infections with hepatitis E virus of genotype 4 in Europe. Intervirology 2014, 57, 43-48. [CrossRef]

9. Aspinall, E.J.; Couturier, E.; Faber, M.; Said, B.; Ijaz, S.; Tavoschi, L.; Takkinen, J.; Adlhoch, C. The Country Experts. Hepatitis E virus infection in Europe: Surveillance and descriptive epidemiology of confirmed cases, 2005 to 2015. Euro Surveill. 2017, 22, 30561. [CrossRef]

10. Lhomme, S.; Gallian, P.; Dimeglio, C.; Assal, A.; Abravanel, F.; Tiberghien, P.; Izopet, J. Viral load and clinical manifestations of hepatitis E virus genotype 3 infections. J. Viral Hepat. 2019, 26, 1139-1142. [CrossRef]

11. Abravanel, F.; Dimeglio, C.; Castanier, M.; Péron, J.M.; Kamar, N.; Lhomme, S.; Izopet, J. Does HEV-3 subtype play a role in the severity of acute hepatitis E? Liver Int. 2020, 40, 333-337. [CrossRef] [PubMed]

12. Lee, G.H.; Tan, B.H.; Teo, E.C.; Lim, S.G.; Dan, Y.Y.; Wee, A.; Aw, P.P.; Zhu, Y.; Hibberd, M.L.; Tan, C.K.; et al. Chronic Infection With Camelid Hepatitis E Virus in a Liver Transplant Recipient Who Regularly Consumes Camel Meat and Milk. Gastroenterology 2016, 150, 355-357.e3. [CrossRef] [PubMed]

13. Woo, P.C.; Lau, S.K.; Teng, J.L.; Cao, K.Y.; Wernery, U.; Schountz, T.; Chiu, T.H.; Tsang, A.K.; Wong, P.C.; Wong, E.Y.; et al. New Hepatitis E Virus Genotype in Bactrian Camels, Xinjiang, China, 2013. Emerg Infect Dis. 2016, 22, 2219-2221. [CrossRef] [PubMed]

14. Sridhar, S.; Yip, C.C.; Wu, S.; Chew, N.F.; Leung, K.H.; Chan, J.F.; Zhao, P.S.; Chan, W.M.; Poon, R.W.; Tsoi, H.W.; et al. Transmission of Rat Hepatitis E Virus Infection to Humans in Hong Kong: A Clinical and Epidemiological Analysis. Hepatology 2020. [CrossRef] [PubMed]

15. Adlhoch, C.; Baylis, S.A. The emergence of zoonotic rat hepatitis E virus infection. Hepatology 2020, 72, 1155. [CrossRef] 
16. Wedemeyer, H.; Pischke, S.; Manns, M.P. Pathogenesis and treatment of hepatitis e virus infection. Gastroenterology 2012, 142, 1388-1397.e1. [CrossRef]

17. European Association for the Study of the Liver. EASL Clinical Practice Guidelines on hepatitis E virus infection. J. Hepatol. 2018, 68, 1256-1271. [CrossRef]

18. Kamar, N.; Garrouste, C.; Haagsma, E.B.; Garrigue, V.; Pischke, S.; Chauvet, C.; Dumortier, J.; Cannesson, A.; Cassuto-Viguier, E.; Thervet, E.; et al. Factors associated with chronic hepatitis in patients with hepatitis E virus infection who have received solid organ transplants. Gastroenterology 2011, 140, 1481-1489. [CrossRef]

19. Vollmer, T.; Diekmann, J.; Eberhardt, M.; Knabbe, C.; Dreier, J. Hepatitis E in blood donors: Investigation of the natural course of asymptomatic infection, Germany, 2011. Euro Surveill. 2016, 21, 30332. [CrossRef]

20. Zafrullah, M.; Khursheed, Z.; Yadav, S.; Sahgal, D.; Jameel, S.; Ahmad, F. Acidic pH enhances structure and structural stability of the capsid protein of hepatitis E virus. Biochem. Biophys. Res. Commun. 2004, 313, 67-73. [CrossRef]

21. Halfon, P.; Khiri, H.; Gerolami, V.; Bourliere, M.; Feryn, J.M.; Reynier, P.; Gauthier, A.; Cartouzou, G. Impact of various handling and storage conditions on quantitative detection of hepatitis C virus RNA. J. Hepatol. 1996, 25, 307-311. [CrossRef]

22. Myint, K.S.; Endy, T.P.; Shrestha, M.P.; Shrestha, S.K.; Vaughn, D.W.; Innis, B.L.; Gibbons, R.V.; Kuschner, R.A.; Seriwatana, J.; Scott, R.M. Hepatitis E antibody kinetics in Nepalese patients. Trans. R Soc. Trop. Med. Hyg. 2006, 100, 938-941. [CrossRef] [PubMed]

23. Wen, G.P.; Tang, Z.M.; Yang, F.; Zhang, K.; Ji, W.F.; Cai, W.; Huang, S.J.; Wu, T.; Zhang, J.; Zheng, Z.Z.; et al. A valuable antigen detection method for diagnosis of acute hepatitis E. J. Clin. Microbiol. 2015, 53, 782-788. [CrossRef] [PubMed]

24. Matheus, S.; Deparis, X.; Labeau, B.; Lelarge, J.; Morvan, J.; Dussart, P. Discrimination between primary and secondary dengue virus infection by an immunoglobulin $\mathrm{G}$ avidity test using a single acute-phase serum sample. J. Clin. Microbiol. 2005, 43, 2793-2797. [CrossRef]

25. Levett, P.N.; Sonnenberg, K.; Sidaway, F.; Shead, S.; Niedrig, M.; Steinhagen, K.; Horsman, G.B.; Drebot, M.A. Use of immunoglobulin $\mathrm{G}$ avidity assays for differentiation of primary from previous infections with West Nile virus. J. Clin. Microbiol. 2005, 43, 5873-5875. [CrossRef]

26. Grangeot-Keros, L.; Mayaux, M.J.; Lebon, P.; Freymuth, F.; Eugene, G.; Stricker, R.; Dussaix, E. Value of cytomegalovirus (CMV) IgG avidity index for the diagnosis of primary CMV infection in pregnant women. J. Infect. Dis. 1997, 175, 944-946. [CrossRef]

27. Bigaillon, C.; Tessé, S.; Lagathu, G.; Nicand, E. Use of hepatitis E IgG avidity for diagnosis of hepatitis E infection. J. Virol. Methods. 2010, 164, 127-130. [CrossRef]

28. Bendall, R.; Ellis, V.; Ijaz, S.; Thurairajah, P.; Dalton, H.R. Serological response to hepatitis E virus genotype 3 infection: IgG quantitation, avidity, and IgM response. J. Med. Virol. 2008, 80, 95-101. [CrossRef]

29. De Sabato, L.; Di Bartolo, I.; Lapa, D.; Capobianchi, M.R.; Garbuglia, A.R. Molecular Characterization of HEV Genotype 3 in Italy at Human/Animal Interface. Front. Microbiol. 2020, 11, 137. [CrossRef] [PubMed]

30. La Rosa, G.; Muscillo, M.; Vennarucci, V.S.; Garbuglia, A.R.; La Scala, P.; Capobianchi, M.R. Hepatitis E virus in Italy: Molecular analysis of travel-related and autochthonous cases. J. Gen. Virol. 2011, 92, 1617-1626. [CrossRef]

31. Kumar, S.; Stecher, G.; Li, M.; Knyaz, C.; Tamura, K. MEGA X: Molecular Evolutionary Genetics Analysis across Computing Platforms. Mol. Biol. Evol. 2018, 35, 1547-1549. [CrossRef] [PubMed]

32. Riveiro-Barciela, M.; Rando-Segura, A.; Barreira-Díaz, A.; Bes, M.P.; Ruzo, S.; Piron, M.; Quer, J.; Sauleda, S.; Rodríguez-Frías, F.; Esteban, R.; et al. Unexpected long-lasting anti-HEV IgM positivity: Is HEV antigen a better serological marker for hepatitis $\mathrm{E}$ infection diagnosis? J. Viral Hepat. 2020, 27, 747-753. [CrossRef]

33. Vollmer, T.; Knabbe, C.; Dreier, J. Comparison of real-time PCR and antigen assays for detection of hepatitis E virus in blood donors. J. Clin. Microbiol. 2014, 52, 2150-2156. [CrossRef] [PubMed]

34. Mishra, S.; Borkakoti, J.; Kumar, S.; Kar, P. Role of HEV antigen detection in HEV-related acute viral hepatitis and acute liver failure. J. Med. Virol. 2016, 88, 2179-2185. [CrossRef] [PubMed]

35. Zhao, C.; Geng, Y.; Harrison, T.J.; Huang, W.; Song, A.; Wang, Y. Evaluation of an antigen-capture EIA for the diagnosis of hepatitis E virus infection. J. Viral Hepat. 2015, 22, 957-963. [CrossRef] [PubMed]

36. Trémeaux, P.; Lhomme, S.; Chapuy-Regaud, S.; Peron, J.M.; Alric, L.; Kamar, N.; Izopet, J.; Abravanel, F. Performance of an antigen assay for diagnosing acute hepatitis E virus genotype 3 infection. J. Clin. Virol. 2016, 79, 1-5. [CrossRef] 